\title{
The aqueous extract of Chinese medicinal herb Brucea javanica suppresses the growth of human liver cancer and the derived stem-like cells by
} apoptosis

\author{
This article was published in the following Dove Press journal: \\ Drug Design, Development and Therapy \\ 17 June 2016 \\ Number of times this article has been viewed
}

\author{
Jian-He Chen* \\ Seung-Hun Kim* \\ Po-Wei Fan \\ Chun-Yen Liu \\ Chang-Hung Hsieh \\ Kang Fang \\ Department of Life Science, National \\ Taiwan Normal University, Taipei, \\ Taiwan \\ *These authors contributed equally \\ to this work
}

\begin{abstract}
Being effective and relatively safe, the traditional Chinese medicinal herb Brucea javanica (BJ) has been valuable in curing patients in East Asia and its nearby regions for years. Recent reports suggested that the medicinal herb possesses broad antitumor activity against various cancer cells. This study evaluated whether low concentrations of BJ aqueous extract inhibited the growth of liver cancer cells. Experiments including flow cytometry and Western blot analysis established the development of apoptotic cell death after treatment. Further experiments evaluated the growth of the enriched spheroids. BJ not only reduced the expression of stem cell markers but also eliminated tumor spheroids by apoptotic death. The findings suggest $\mathrm{BJ}$ is a promising supplement to the current therapy regimen and highlight the opportunity of $\mathrm{BJ}$ as a practical avenue to suppress the growth of the stem cells in liver cancer.
\end{abstract}

Keywords: Brucea javanica, apoptosis, stem cells, human liver cancer cells

\section{Introduction}

Recent active research has revealed that Chinese herbal medicine (CHM) has advanced for clinical use by identifying potent extracts and their active ingredients. ${ }^{1,2}$ Among them, the aqueous extract of the medicinal plant Brucea javanica $(\mathrm{BJ})$ exhibited therapeutic efficacy, and it was clinically useful. ${ }^{3,4}$ As an effective and a relatively safe traditional Chinese herb, the medicine has cured patients suffering from amebic dysentery, malaria, and various parasites in East Asia regions for years. ${ }^{4,5}$ The emulsified formula of the seeds of BJ has been approved and is commercially available in oriental countries to treat various types of cancer, such as lung cancer diagnosed at middle or late stage. ${ }^{6}$ The chemopreventive BJ fruit extract exhibited cytotoxic activity in pancreatic cancer cells. ${ }^{7}$ With the combination of conventional chemotherapy, BJ oil emulsion injection offered a safe and an efficient regimen in treating patients with advanced gastric cancer and lung adenocarcinoma. ${ }^{8,9}$ Moreover, the ethyl acetate extract of BJ seeds was also applied to treat inflammatory diseases. ${ }^{10}$

Hepatocellular carcinomas rank as the fifth most common malignancy worldwide, and the incidence grows continuously because of poor prognosis. ${ }^{11}$ Several herbal compounds and their composite formulas are well known and effective in both in vitro and in vivo models. ${ }^{12}$ Despite reports on BJ as a valid cancer treatment, ${ }^{13,14}$ whether or not its aqueous extract is useful against human liver cancer and the progenitor stem-like cells remained unknown. To address this topic, this study reported that low concentrations
Correspondence: Kang Fang

Department of Life Science, National Taiwan Normal University, 88 Ting-Chou Road, Sector 4, Taipei II6, Taiwan

Tel +8862 77346293

Fax +8862293I 2904

Email kangfang@ntnu.edu.tw 
of aqueous extract of BJ not only inhibited the growth of liver cancer cells but also blocked tumor-initiating capacity of the derived spheroids. The developed apoptotic cell death contributed to the reduced cell growth. Current therapies tend to survive residual stem-like cells that subsequently develop resistant clones and result in relapse in patients. The findings indicated additional values of BJ extract in restraining propagation of liver cancer stem-like cells. The promising application highlights the importance of CHM to overcome the resistance caused by residual stem cells. The discovery complements the conventional cancer treatment.

\section{Materials and methods}

\section{Cell culture}

Human hepatocellular carcinoma cell lines, HepG2 (HB-8065, wild-type p53) and Hep3B (HB-8064, p53-null) were acquired from American Type Culture Collection (ATCC, Manassas, VA, USA). Huh7 (mutant p53) cells were from Japanese Collection of Research Bioresources. Both HepG2 and Huh7 cells were cultured in Dulbecco's Modified Eagle's Medium (DMEM). The Hep3B cells were cultured in RPMI (Roswell Park Memorial Institute)-1640 media. The cultures were supplemented with L-glutamine, sodium pyruvate, and $10 \%$ fetal bovine serum at $37^{\circ} \mathrm{C}$ in a humidified atmosphere with $5 \% \mathrm{CO}_{2}$. The cell media was replaced every 3 days or 4 days and the subcultured cells that reached $80 \%-90 \%$ confluence were used for experiments. This study was approved by the institutional ethics committee of National Taiwan Normal University.

\section{Chemicals and reagents}

Sun Ten Pharmaceutical (Taichung, Taiwan) provided the aqueous extract of the whole BJ plants. The collected samples that were cut into thin sections with razor blade were mixed with sterile water. The mixture was pulverized using a sterile mortar and pestle. The final solution was placed in a sterile centrifuge tube, boiled, cooled, shaken gently at $4{ }^{\circ} \mathrm{C}$ overnight, and centrifuged at $15,000 \times g$ for 10 minutes to separate pellet solids. The supernatant was then withdrawn, placed in a sterile vial, diluted with boiled water to make a final concentration of $1 \mathrm{~g} / \mathrm{mL}$, and stored at $4^{\circ} \mathrm{C}$. The waterdiluted supernatant was used for the study.

The chemicals including propidium iodide (PI), trypan blue, Tris- $\mathrm{HCl}$, and Triton $\mathrm{X}-100$ were from purchased Sigma-Aldrich Co. (St Louis, MO, USA), and penicillinstreptomycin, fetal bovine serum, glutamine, trypsin-sodium ethylenediaminetetraacetate, and DMEM were obtained from Thermo Fisher Scientific (Waltham, MA, USA). Polycarbonate filters were from Millipore (Billerica, MA, USA). The target therapy drug, erlotinib, was from OSI Pharmaceuticals (Melville, NY, USA).

\section{Cell viability assay}

The cell viability was determined by 3-[4, 5-dimethylthiazol2-yl]-2, 5 diphenyl tetrazolium bromide (MTT) cell viability assay. Briefly, a total of $5 \times 10^{3}$ cells were planted in a 96-well plate and then incubated with different concentrations of BJ for times as indicated. Solution containing MTT $(0.5 \mathrm{mg} / \mathrm{mL}$; Sigma-Aldrich Co.) was added and incubated for 3 hours. Dimethyl sulfoxide was added to dissolve the crystals. The absorption at $570 \mathrm{~nm}$ with $630 \mathrm{~nm}$ as the reference wavelength was measured by a Microplate ELISA Reader. Relative cell viability was calculated as the percentage of the control. All cell viability assays were performed in triplicate and repeated in three independent experiments.

\section{Flow cytometry}

Cells were analyzed using FACS Calibur ${ }^{\mathrm{TM}}$ flow cytometer (BD Biosciences, San Jose, CA, USA) to determine cell cycle distribution. A total of $3 \times 10^{6}$ cells were treated with various concentrations of aqueous extractions of BJ for the periods as indicated. For sample preparation, both medium and the trypsinized cells were centrifuged and the supernatant was removed. The collected cells washed with phosphate-buffered saline (PBS) were treated with alcohol and PBS mixture $(7: 3)$ at $-20^{\circ} \mathrm{C}$ for 24 hours. Right before analysis, the sample cells were labeled with $10 \mu \mathrm{g} / \mathrm{mL}$ PI (Sigma-Aldrich Co.) and dissolved in PBS containing $10 \mu \mathrm{g} /$ mL RNase A (ICN Pharmaceutical, Costa Mesa, CA, USA) for 30 minutes in darkness. Data were analyzed by FlowJo software (Tree Star, Ashland, OR, USA).

\section{Determination of apoptosis by double staining with Annexin V-fluorescein isothiocyanate and $\mathrm{PI}$}

Cells seeded in 12 -well plates at $2 \times 10^{5}$ cells/well for 16 hours were treated with various concentrations of BJ extract or water control and incubated at $37^{\circ} \mathrm{C}$ for 12 hours. The gathered cells were labeled with the mixture of $1: 1$ ratio Annexin V/fluorescein isothiocyanate $(20 \mu \mathrm{g} / \mathrm{mL})$ and PI $(50 \mu \mathrm{g} / \mathrm{mL})$ at room temperature for 30 minutes in darkness. The flow cytometer FACS Calibur ${ }^{\mathrm{TM}}$ was employed to determine the early and late phases of apoptotic cell distribution, and the data were analyzed by FlowJo software.

\section{Cell lysates for Western blotting analysis}

Cells plated $\left(1 \times 10^{6}\right.$ per well $)$ in serum-free media were treated with various concentrations of aqueous extract of BJ for 
12 hours. The collected medium was centrifuged to remove cell debris before being lysed. The protein concentrations of the lysates were determined using a Bio-Rad protein assay kit (Bio-Rad Laboratories Inc., Hercules, CA, USA) with bovine serum albumin (BSA) as the standard. The lysates resolved by electrophoresis were transferred to nitrocellulose membrane. The blotted membrane blocked with $2 \%$ skim milk for 30 minutes at room temperature was incubated overnight at $4{ }^{\circ} \mathrm{C}$ with the primary antibodies of $1: 2,000$ dilution in PBS- or Tris-buffered saline supplemented with $0.05 \%$ Tween-20. The blots were incubated with appropriate secondary antibodies that conjugated with horseradish peroxidase and visualized with enhanced chemiluminescence Western blotting substrate (Pierce, Rockford, IL, USA). The signals were recorded with enhanced LAS-4000 (FUJIFILM) apparatus, and the band intensities of images were analyzed by ImageJ software.

\section{Enrichment of spheroids}

Human liver carcinoma Hep3B cells were enriched to acquire enough target spheroid cells. To support growing undifferentiated spheroids, cells were cultured at $1 \times 10^{4}$ cells $/ \mathrm{mL}$ in serum-free DMEM-F12 (Thermo Fisher Scientific). The media in ultra-low attachment flasks (Corning Incorporated, Corning, NY, USA) contained $50 \mu \mathrm{g} / \mathrm{mL}$ of insulin (SigmaAldrich Co.), 0.4\% albumin bovine fraction V (SigmaAldrich Co.), N-2 Plus Media Supplement (R\&D Systems, Inc., Minneapolis, MN, USA), B-27 supplement (Thermo Fisher Scientific), $20 \mathrm{ng} / \mathrm{mL}$ epidermal growth factor (EGF; PeproTech, Rocky Hill, NJ, USA), $10 \mathrm{ng} / \mathrm{mL}$ basic fibroblast growth factor (PeproTech), and heparin $(2 \mu \mathrm{g} / \mathrm{mL})$. Floating sphere cultures were expanded by mechanical dissociation, followed by replating of single cells in complete fresh medium every 3 days. For clonal expansion, single cells were added to each well of 96-well ultra-low attachment tissue culture plates (Corning Incorporated) and clonal expansion monitored at the indicated time points. The collected spheroid cells were subject to experiments in non-adherent culture conditions. Spheroid diameters were measured using Image-Pro plus 6.3 (Media Cybernetics, Bethesda, MD, USA).

\section{Colony formation assay}

The spheroid growth was determined by colony formation assay. A total of 200 cells/well in 12-well ultra-low attachment tissue culture plates were grown in growth-factorsupplemented medium for 8 days. When cell clones reached to $>50$ cells, the spheres were treated with various concentrations of BJ extract for 24 hours. Then, the spheroid mixtures were cultured in soft agar with $0.5 \%$ agarose on top and $0.3 \%$ agar on bottom layers. After 28 days, the plates were stained with $0.002 \%$ crystal violet and incubated at $37^{\circ} \mathrm{C}$ for 18 hours. Following the removal of the dye solution, colonies with more than 50 cells were counted as positive. Three individual experiments were conducted for each condition.

\section{Bromodeoxyuridine incorporation of spheroids}

The assay to measure the proliferation of spheres used bromodeoxyuridine (BrdU) Labeling and Detection Kit II (Hoffman-La Roche Ltd., Basel, Switzerland). The spheroids treated with BJ extract at various concentrations were incubated in media containing $10 \mu \mathrm{M}$ BrdU for 1 hour. The fixatives with 3:7 mixture of $50 \mathrm{mM}$ glycine solution ( $\mathrm{pH} 2.0$ ) and $70 \mu \mathrm{L}$ ethanol were added and incubated overnight at $-20^{\circ} \mathrm{C}$. The spheres were washed with PBS and incubated at $37^{\circ} \mathrm{C}$ in solution containing diluted anti-BrdU antibody (1:1,000 dilution) for 30 minutes. The cells were suspended in $1 \%$ BSA containing Alexa Fluor 488-conjugated secondary antibody (1:250 dilution) for 2 hours in darkness. An inverted fluorescence microscope was used to record the fluorescence images.

\section{Terminal deoxynucleotidyl transferase- mediated deoxyuridine triphosphate (dUTP) nick-end labeling assay of spheroids}

The apoptotic death of spheroids was evaluated by terminal deoxynucleotidyl transferase-mediated dUTP nick-end labeling (TUNEL) staining. Cells treated with various concentrations of BJ extract were permeabilized in $10 \mathrm{mM}$ citrate buffer, $\mathrm{pH}$ 6.0. After blocking the nonspecific labeling with PBS containing 2\% BSA and 0.5\% NP40, cells were incubated in a TUNEL reaction solution mixed with $9 \mathrm{mM}$ dUTP, 1 mM digoxigenin-labeled dUTP (Hoffman-La Roche Ltd.), $2.5 \mathrm{mM}$ cobalt chloride, $100 \mathrm{mM}$ Tris $\mathrm{pH}$ 7.6, and $0.3 \mathrm{U} / \mu \mathrm{L}$ terminal deoxynucleotidyl transferase. After 1 -hour treatment at $37^{\circ} \mathrm{C}$ in a humidified environment, the sections were washed with PBS, incubated with a 1:200 dilution of digoxigenin antibody conjugated with horseradish peroxidase (Hoffman-La Roche Ltd.). With the removal of unbound antibody, cells were observed by fluorescence microscope. Positively stained nuclei with 50 spheroidal sections were counted. Five slides of each concentration and control sections per condition were observed for each experiment, and three independent experiments carried out. All colored images were converted to black and white ones by Adobe Photoshop software. The quantitation of visualized TUNEL was performed by ImageJ software (http://rsbweb.nih.gov/). 


\section{Statistical analysis}

All the data were presented as mean \pm SD from three individual experiments. Statistical analysis used Student's $t$-test. All statistical analyses were performed using GraphPad Prism 5.00 for Windows (GraphPad Software, Inc., La Jolla, CA, USA). Statistical differences were considered significant with $P<0.05$.

\section{Results}

\section{Low concentrations of $B$ J aqueous extract inhibited the growth of liver cancer cells}

The determination of viability changes in different liver cancer cells used aqueous extract of BJ with concentrations ranging from $0.1 \mathrm{mg} / \mathrm{mL}$ to $1.0 \mathrm{mg} / \mathrm{mL}$. Among the cells studied, the growth of Hep3B cells was reduced and the effect dose dependent with a half maximal inhibitory concentration $\left(\mathrm{IC}_{50}\right)$ of $0.2 \mathrm{mg} / \mathrm{mL}$ as indicated by MTT assay (Figure 1A). Within the concentrations studied, there was no apparent growth inhibition in HepG2 and Huh7 cells.

\section{$B J$ increased sub-G, population} distribution in Hep3B cells

The aqueous extract exhibited cytotoxicity in Hep3B cells when the concentration reached $0.2 \mathrm{mg} / \mathrm{mL}$. Thus, all experiments used BJ extract of $0.1 \mathrm{mg} / \mathrm{mL}, 0.2 \mathrm{mg} / \mathrm{mL}, 0.5 \mathrm{mg} /$ $\mathrm{mL}$, and $1 \mathrm{mg} / \mathrm{mL}$. Flow cytometry analysis of PI-stained Hep3B cells revealed the appearance of sub- $\mathrm{G}_{1}$ cell populations and the effect was concentration dependent (Figure 1B), while both HepG2 and Huh7 cells remained unchanged. Furthermore, Hep3B cells exposed to different concentrations of extract underwent flow cytometry analysis following Annexin V and PI double staining. The early and late apoptotic phase populations in Hep3B cells rose to $15 \%$ and $27 \%$, respectively, when treated with $1.0 \mathrm{mg} / \mathrm{mL}$ of BJ extract for 12 hours (Figure $1 \mathrm{C}$ and D). The results altogether proved that the apoptotic cell death by aqueous extract contributed to the reduced viable cells under the influence of BJ extract.

\section{BJ extract enhanced apoptotic characteristics in Hep3B cells}

To ensure that BJ induced apoptosis, the assessment on cytochrome $c$ release from mitochondria was visualized by confocal microscopy analysis. The appearance of coalesced green mitochondria marker and red cytochrome $c$ suggested that, in Hep3B cells, BJ extract induced the release of cytochrome $c$ from mitochondria after 6 hours of treatment and the effect was concentration dependent (Figure 2). The released cytochrome $c$ forestalls apoptosis that predates progressive cell death in Hep3B cells.

The collected protein lysates from HepG2, Huh7, and Hep3B cells treated with various concentrations of BJ extract for 12 hours were subjected to Western blot analysis. $\mathrm{BJ}$ induced dose-dependent attenuation of EGF receptor (EGFR) and Akt in Hep3B cells and inhibited phosphorylation of $\mathrm{Akt}^{\mathrm{S} 473}$ (Figure 3). The concomitant cleavage of poly(adenosine diphosphate-ribose)polymerase (PARP), dissipation of procaspase-9 and procaspase-3, and the developed active caspase- 3 fragment set the stage of apoptotic death in Hep3B cells. The increased concentrations of BJ extract did not affect HepG2 or Huh7 cells.

\section{BJ extract mitigated growth rates and induced apoptosis in Hep3B spheroids}

To enrich and isolate stem-like cells, the adherent Hep3B cells were propagated in serum-free medium and supplemented with various growth factors. The evolved three-dimensional clusters grew in suspension as spheroid. A comparable higher concentration of BJ extract was necessary to suppress the growth of Hep3B spheroids as proved by sphere formation assay. Compared to vehicle control, tumor sphere sizes became smaller with increased extract concentrations and extended time points (Figure 4A). Experiments of soft agar assay which were seeded with drug-treated spheres and incubated for 28 days supported the observation. The reduced colony sizes (Figure 4B) and decreased cluster numbers (Figure 4C) suggested that BJ extract is effective in abrogating cancer stem cell (CSC) properties of Hep3B spheroids in comparison with those of HepG2 and Huh7. The inhibitory BJ extract concentration $\mathrm{IC}_{50}$ for Hep3B spheroids was determined as $4 \mathrm{mg} / \mathrm{mL}$ by soft agar assay (Figure 4D).

BJ extract induced apoptotic cell death in the suspension culture cells. More experiment using BrdU incorporation supported the notion that Hep3B required relatively higher concentrations of BJ to impede sphere growth (Figure 5A and B). The emergent apoptosis as evidenced by TUNEL assay (Figure 5C and D) contributed to growth attenuation of the spheroids.

\section{The aqueous $B$ J extract suppressed self-renewal properties and enhanced apoptosis features in Hep3B spheroids}

The suspended spheres developed in serum-free medium were exposed to $2.0 \mathrm{mg} / \mathrm{mL}, 4.0 \mathrm{mg} / \mathrm{mL}$, and $6.0 \mathrm{mg} / \mathrm{mL}$ of BJ extract, respectively, for 12 hours. The depleted intensities of 


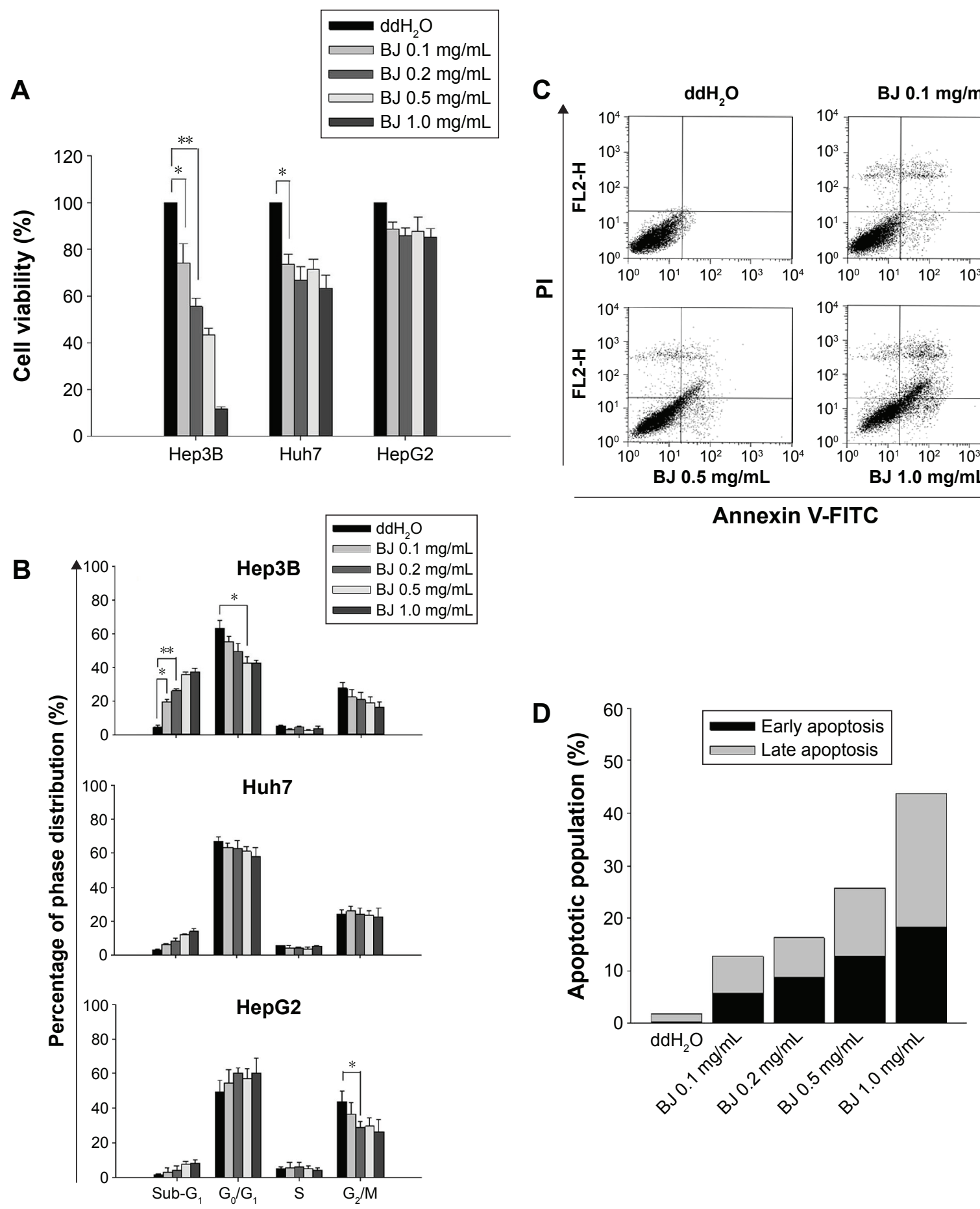

Figure I Dose-response curves of BJ extract in liver cancer cell lines.

Notes: (A) BJ inhibited growth of Hep3B cells. Liver cancer cells Huh7, Hep3B, and HepG2 were cultured in 96-well plate for 28 hours, then treated with various concentrations $(0.1 \mathrm{mg} / \mathrm{mL}, 0.2 \mathrm{mg} / \mathrm{mL}, 0.5 \mathrm{mg} / \mathrm{mL}$, and $1.0 \mathrm{mg} / \mathrm{mL})$ of aqueous BJ extract or water for 12 hours, and stained with MTT. The cell viabilities were converted from the absorbance of drug-treated cells against that of water control. The relative viabilities were obtained by conversion into percentages compared with water treatment control as $100 \%$. The data are the averages of quadruplicate. The results were represented as mean \pm SD of three independent experiments. $* P<0.05$ and $* * P<0.0$ I. (B) $B J$ induced sub-G, populations. Hep3B, Huh7, and HepG2 cells cultured in I\% serum-supplemented RPMI- 1640 were incubated with various concentrations of BJ (0.I mg/mL, $0.2 \mathrm{mg} / \mathrm{mL}, 0.5 \mathrm{mg} / \mathrm{mL}$ and $1.0 \mathrm{mg} / \mathrm{mL}$ ) and water control for 12 hours before being labeled with PI followed by flow cytometry analysis. (C) Two-dimensional flow analysis. Hep3B cells were treated with BJ $(0.1 \mathrm{mg} / \mathrm{mL}, 0.2 \mathrm{mg} / \mathrm{ml}, 0.5 \mathrm{mg} / \mathrm{mL}$, and $1.0 \mathrm{mg} / \mathrm{mL})$ or water for 12 hours and the trypsinized cells were analyzed by Annexin V/PI-stained flow cytometry. (D) Quantitative analysis of Annexin V-positive cell populations. Hep3B cells were treated with various concentrations $(0.1 \mathrm{mg} / \mathrm{mL}, 0.2 \mathrm{mg} / \mathrm{mL}, 0.5 \mathrm{mg} / \mathrm{mL}$, and $1.0 \mathrm{mg} / \mathrm{mL}$ ) of $\mathrm{BJ}$ as indicated for 12 hours and the trypsinized cells were analyzed by flow cytometry. The early (dark) and late (light) apoptotic population distributions were expressed as mean values from three independent experiments.

Abbreviations: B], Brucea javanica; FITC, fluorescein isothiocyanate; MTT, 3-[4, 5-dimethylthiazol-2-yl]-2, 5 diphenyl tetrazolium bromide; PI, propidium iodide; RPMI, Roswell Park Memorial Institute; SD, standard deviation. 


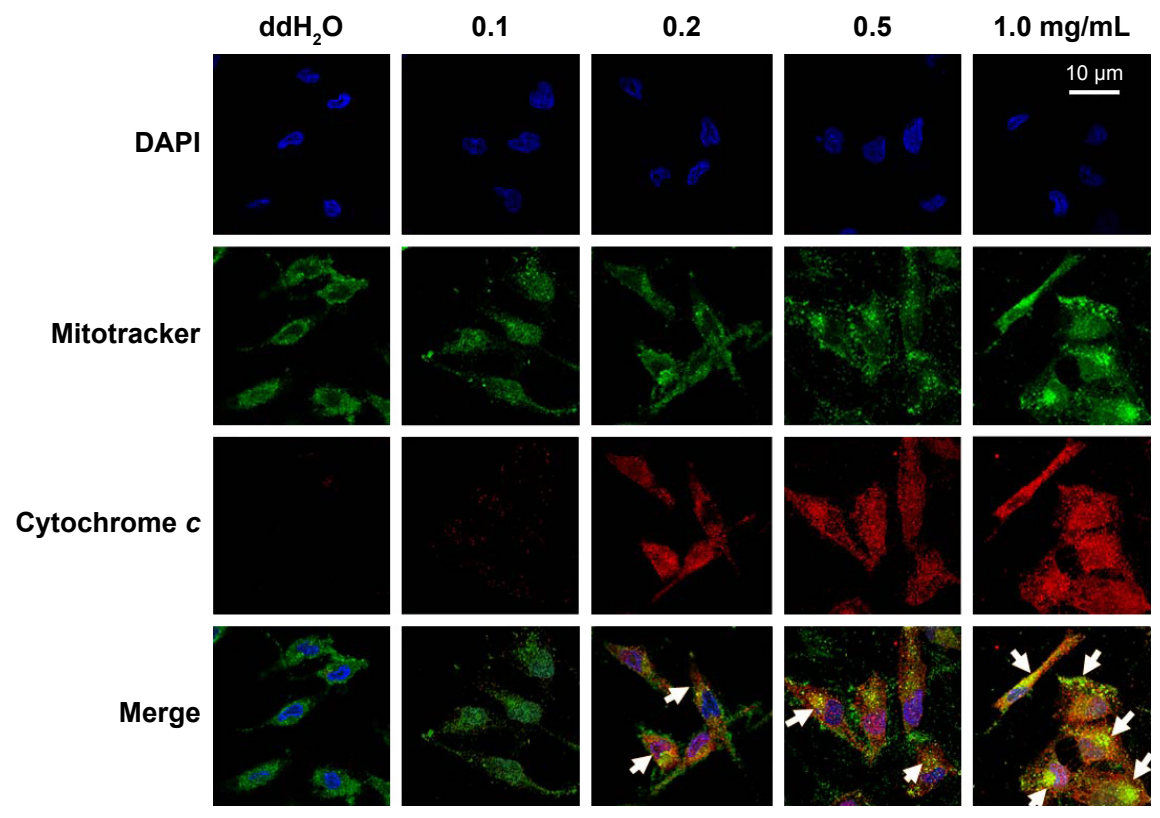

Figure 2 Release of cytochrome $c$ from mitochondria.

Notes: Hep3B cells treated with various concentrations of BJ $(0.1 \mathrm{mg} / \mathrm{mL}, 0.2 \mathrm{mg} / \mathrm{mL}, 0.5 \mathrm{mg} / \mathrm{mL}$, and $1.0 \mathrm{mg} / \mathrm{mL})$ and water control for 6 hours were fixed and incubated with antibody against cytochrome $c$ followed by secondary antibody conjugated with TRITC (red). The slides were counterstained with Mitotracker (green) and DAPI (blue) before being analyzed by confocal microscopy. The arrows indicate the colocalization of red color cytochrome $c$ and green color mitochondria, while blue color indicates nucleus (scale bar $=10 \mu \mathrm{m}$ and applies to all images).

Abbreviations: BJ, Brucea javanica; DAPI, 4',6-diamidino-2-phenylindole; TRITC, tetramethylrhodamine isothiocyanate.
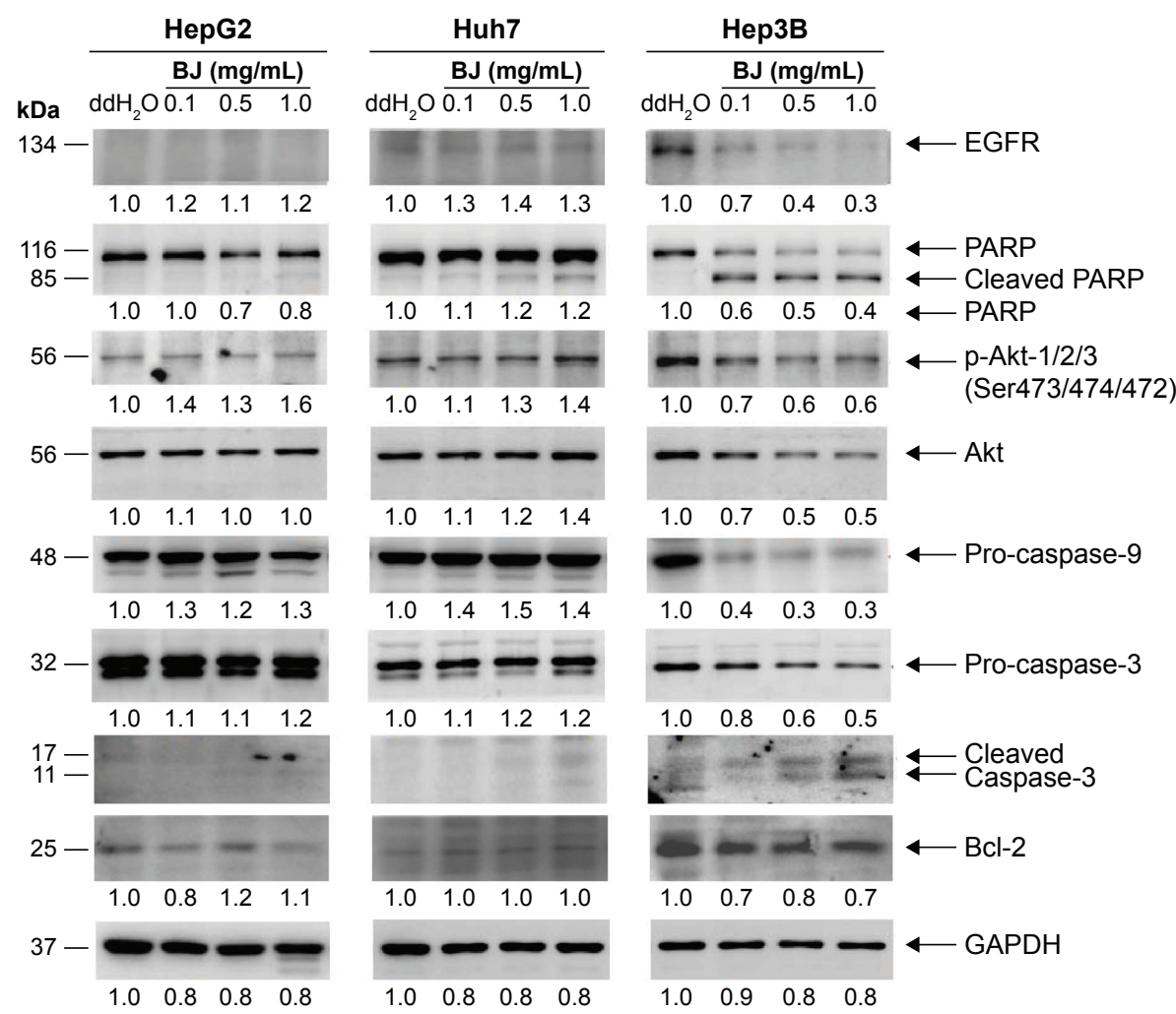

Figure 3 Effects of BJ on apoptotic features in Hep3B cells.

Notes: Liver cancer cells Huh7, Hep3B, and HepG2 cultured in I\% serum-supplemented DMEM or RPMI-I640 and incubated with various concentrations of BJ (0.I mg/mL, $0.5 \mathrm{mg} / \mathrm{mL}$, and $1.0 \mathrm{mg} / \mathrm{mL}$ ) plus vehicle control $\mathrm{H}_{2} \mathrm{O}$ for 12 hours were collected. The protein lysates were conducted for Western blot analysis. The blots were incubated with various primary antibodies, including EGFR, Akt, phosphorylated $\mathrm{Akt}^{\mathrm{s473}}$, caspase-9, caspase-3, Bcl-2, and PARP followed by horseradish peroxidase-conjugated secondary antibodies with GAPDH as loading control. The blots were visualized by ECL staining system. The values shown underneath each blot indicate relative intensities compared with the results of $\mathrm{ddH}_{2} \mathrm{O}$ treatment for each cell line.

Abbreviations: BJ, Brucea javanica; DMEM, Dulbecco's Modified Eagle's Medium; ECL, enhanced chemiluminescence; EGFR, epidermal growth factor receptor; GAPDH, glyceraldehyde 3-phosphate dehydrogenase; PARP, poly(adenosine diphosphate-ribose) polymerase; RPMI, Roswell Park Memorial Institute. 
A

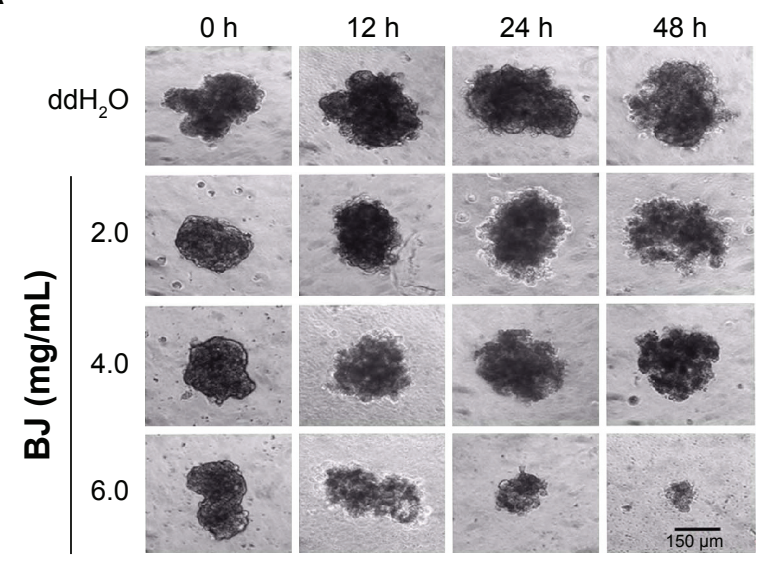

C

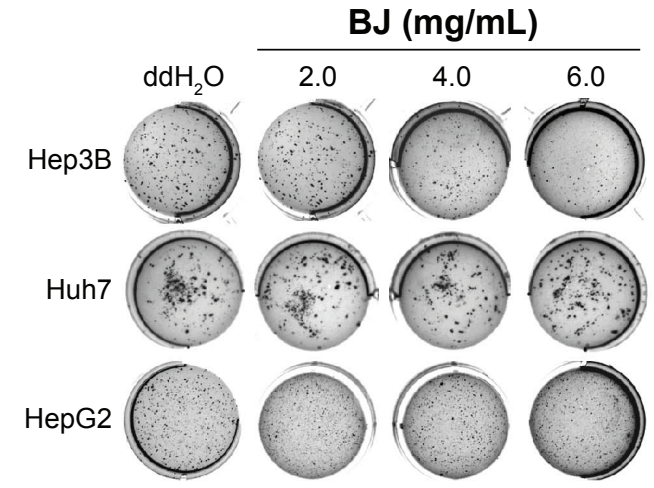

B

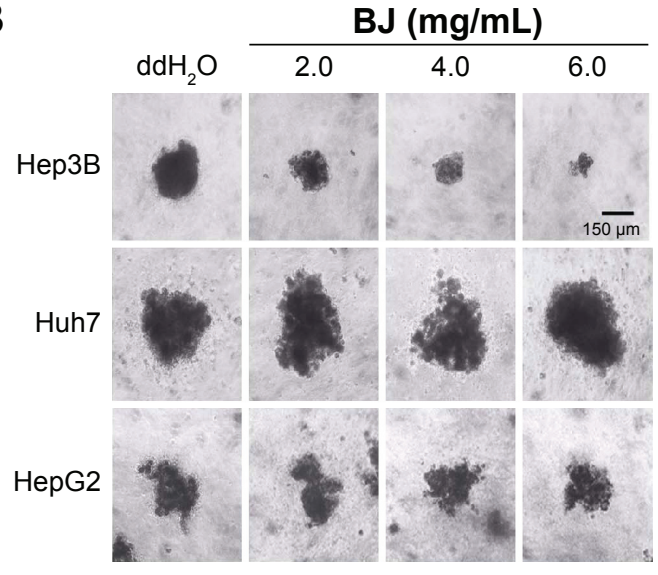

D

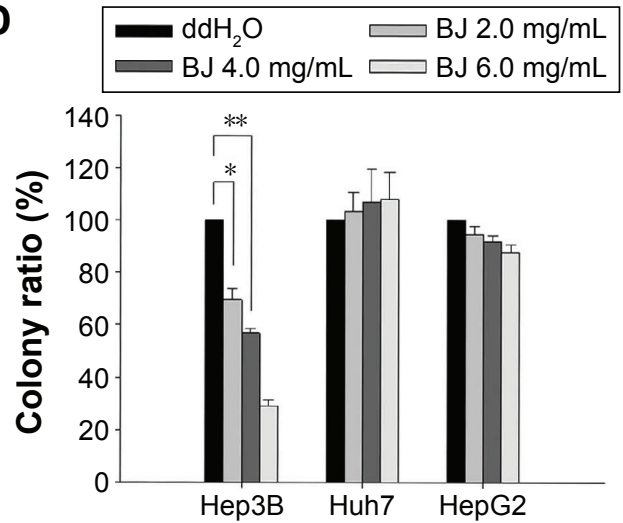

Figure $4 \mathrm{BJ}$ suppressed the growth of Hep3B spheroids.

Notes: (A) Effects of BJ on the growth of multicellular spheroids. Multicellular spheroids were prepared. The representative image of Hep3B spheroid in suspension treated at each condition with $2.0 \mathrm{mg} / \mathrm{mL}, 4.0 \mathrm{mg} / \mathrm{mL}$, and $6.0 \mathrm{mg} / \mathrm{mL}$ of BJ for 12 hours, 24 hours, and 48 hours, respectively, was taken under an inverted microscope (scale bar $=150 \mu \mathrm{m}$ and applies to all images). (B) Representative colony images of soft agar assay. The spheroids derived from Huh7, Hep3B, and HepG2 cells that were treated with $2.0 \mathrm{mg} / \mathrm{mL}, 4.0 \mathrm{mg} / \mathrm{mL}$, and $6.0 \mathrm{mg} / \mathrm{mL}$ of BJ, respectively, for 24 hours, were seeded and grown in soft agar for 28 days and stained with crystal violet. Each representative image of colony grown in soft agar at different concentrations and water control was taken from an inverted microscope (scale bar $=150 \mu \mathrm{m}$ and applies to all images). (C) Soft agar colony forming assay. Soft agar plates of Huh7, Hep3B, and HepG2 spheroids as treated with various concentrations of BJ extract were incubated at $37^{\circ} \mathrm{C}$ for 28 days before being stained with $0.002 \%$ crystal violet. Colonies with $>50$ cells were counted as positive. (D) Statistical analysis. The numbers of colonies of soft agar formation of liver cancer spheroids from Huh7, Hep3B, and HepG2 cells treated with $2.0 \mathrm{mg} / \mathrm{mL}, 4.0 \mathrm{mg} / \mathrm{mL}$, and $6.0 \mathrm{mg} / \mathrm{mL}$ of $B \mathrm{~J} \mathrm{were} \mathrm{counted} \mathrm{and} \mathrm{plotted.} * P<0.05$ and $* * P<0.01$ indicates significant difference between treatment groups and water control as determined from three independent experiments.

Abbreviation: BJ, Brucea javanica.

stemness markers Nanog, CD133, and EpCAM signaled the interruption of self-renewal traits in spheroids with increased BJ extract concentrations. On the other hand, stemness marker ALDH1A1 remained unchanged (Figure 6A). More image analysis showed a gradual decrease in immunofluorescent intensities of Nanog and CD133 in Hep3B spheroids as BJ concentrations were increased. Cleavage of PARP and the emergence of active caspase- 3 fragment confirmed the commencement of apoptotic cell death that diminished the growth of Hep3B spheres as indicated by the attenuated Akt levels (Figure 7A) and EGFR (Figure 7A and B).

\section{Discussion}

New efforts have rejuvenated the CHM research in a hope to broaden the cancer therapy treatment. ${ }^{15}$ Recently, the traditional Chinese medicinal herb BJ has shown promising anticancer potency, ${ }^{14,16}$ and further studies reported that herbal remedy is a promising cancer therapy. ${ }^{4,17}$ In an attempt to exploit more applications of the medicinal herb, we reported that BJ aqueous extract inhibited the self-renewal capacity of the derived human stem-like cells of liver cancer. The findings to overcome the intrinsic resistance in cancer cells implied new and potential values of CHM.

Aqueous extract of $\mathrm{BJ}$ inhibited the proliferation of Hep3B cells with an $\mathrm{IC}_{50}$ of $0.2 \mathrm{mg} / \mathrm{mL}$ of BJ (Figure $1 \mathrm{~A}$ ). The reduced pro-survival signaling Akt supported growth inhibition (Figure 3). Furthermore, the appearance of sub- $\mathrm{G}_{1}$ population (Figure 1B), Annexin V-positive cells (Figure 1C and D), and the release of mitochondrial cytochrome $c$ (Figure 2) indicated the onset of apoptosis. The induced cleavage of procaspase- 3 to active caspase- 3 
A
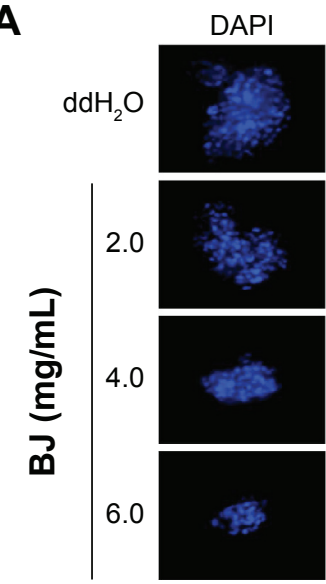

C

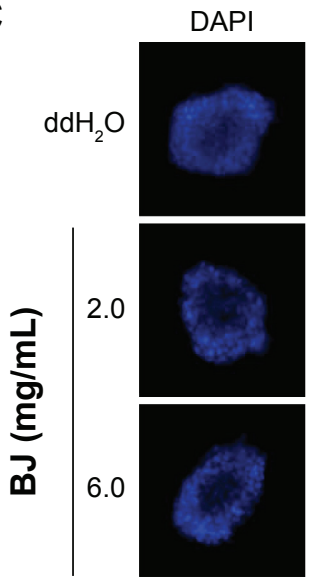

BrdU
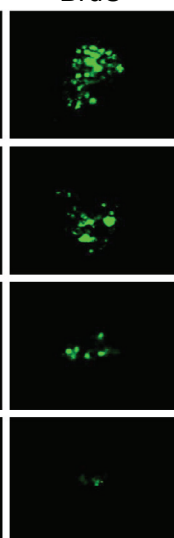

TUNEL
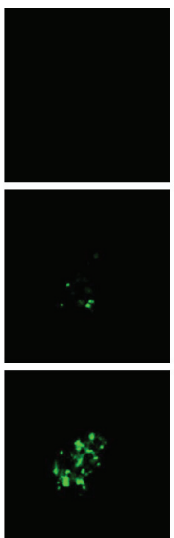
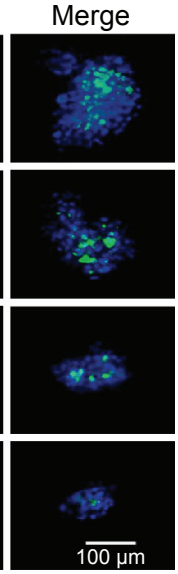

Merge
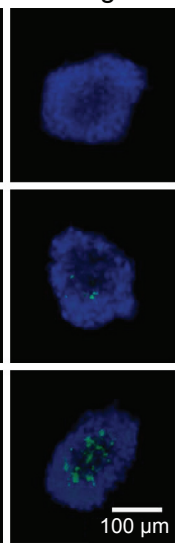

B

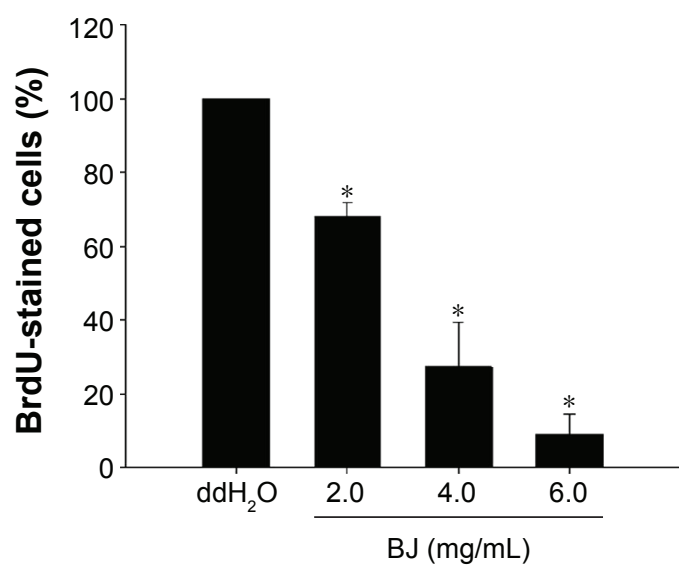

D

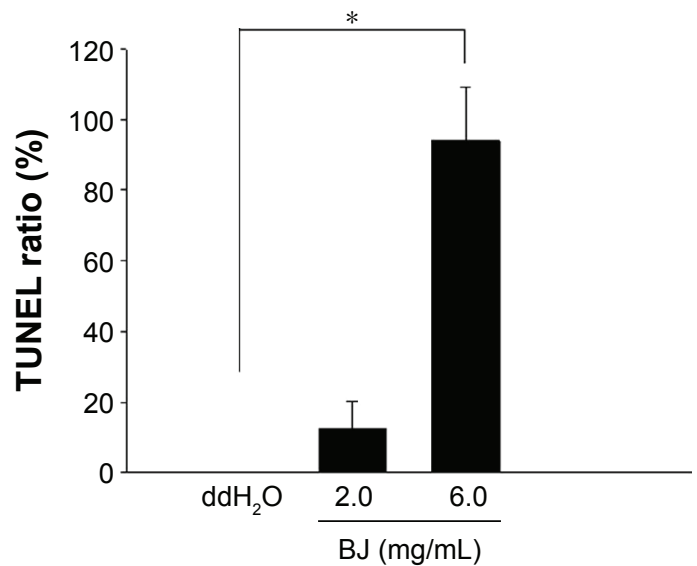

Figure 5 BJ induced apoptotic characteristics in Hep3B spheroids.

Notes: (A) Reduction of BrdU incorporation. Hep3B spheroids were treated with $2.0 \mathrm{mg} / \mathrm{mL}, 4.0 \mathrm{mg} / \mathrm{mL}$, and $6.0 \mathrm{mg} / \mathrm{mL}$ of BJ, respectively, for 24 hours followed by evaluation with BrdU incorporation assay. The merged images of green BrdU and blue nucleus marker DAPI were taken by a fluorescence microscope (scale bar $=100 \mu \mathrm{m}$ and applies to all images). (B) BJ reduced BrdU incorporation. The numbers of fluorescence positive spheres with incorporated BrdU were counted in each field of different concentrations. The numbers as counted were the average of at least three different fields. The data are expressed as mean $\pm S D$ of three individual experiments (*P $<0.05$ ). (C) Dose-dependent increase of TUNEL staining. The spheroids were treated with $2.0 \mathrm{mg} / \mathrm{mL}, 4.0 \mathrm{mg} / \mathrm{mL}$, and $6.0 \mathrm{mg} / \mathrm{mL}$ of BJ, respectively, for 24 hours followed by TUNEL staining evaluation. The merged images of green TUNEL and blue nucleus marker DAPI were taken by a fluorescence microscope (scale bar $=100 \mu \mathrm{m}$ and applies to all images). (D) BJ increased TUNEL positive nuclei. The numbers of fluorescent TUNEL positive spheres in each field of BJ concentration of 2.0 and $6.0 \mathrm{mg} / \mathrm{mL}$ were counted. The numbers counted were the average of at least three different fields. The data are expressed as mean \pm SD of three individual experiments $(* P<0.05)$.

Abbreviations: BJ, Brucea javanica; BrdU, bromodeoxyuridine; DAPI, 4',6-diamidino-2-phenylindole; TUNEL, terminal deoxynucleotidyl transferase-mediated dUTP nickend labeling.

fragment executed apoptosis by cleaving downstream cellular proteins at appointed aspartate residues. ${ }^{18}$ Activated caspases cleaved downstream substrate PARP and triggered final apoptosis. Western blot analysis showed that BJ extract mediated procaspase-3 cleavage. The followed-up PARP cleavage accelerated cell death (Figure 3 ). The enhanced apoptotic characteristics were consistent with the diminished viable cells when concentrations of BJ extract were increased.

More experiments suggested that a relatively higher concentration of aqueous BJ extract is needed to reduce the growth of Hep3B spheroids that were enriched from monolayer cells. To learn more of the inhibition, the increased concentrations of BJ progressively moderated self-renewal capacities of stem-like cells as proved by soft agar (Figure 4A-D) and BrdU assays (Figure 5A and B). The development of apoptosis indicated the elimination of stemlike cells as demonstrated by TUNEL staining (Figure 5C and D) and Western blot analysis. The ultimate emergence of caspase- 3 fragment and cleaved PARP implied the impairment of the progenitor spheroids as seen by the diminished stem cell markers, including CD133, Nanog, and EpCAM. The work established that BJ acts as a complementary therapeutic agent and is capable of overcoming the residual drug resistant stem-like cells.

The results also asserted the significance of EGFR signaling in maintaining survival of Hep3B cells and exposure to BJ abolished the pathway. The findings supported that BJ 
A
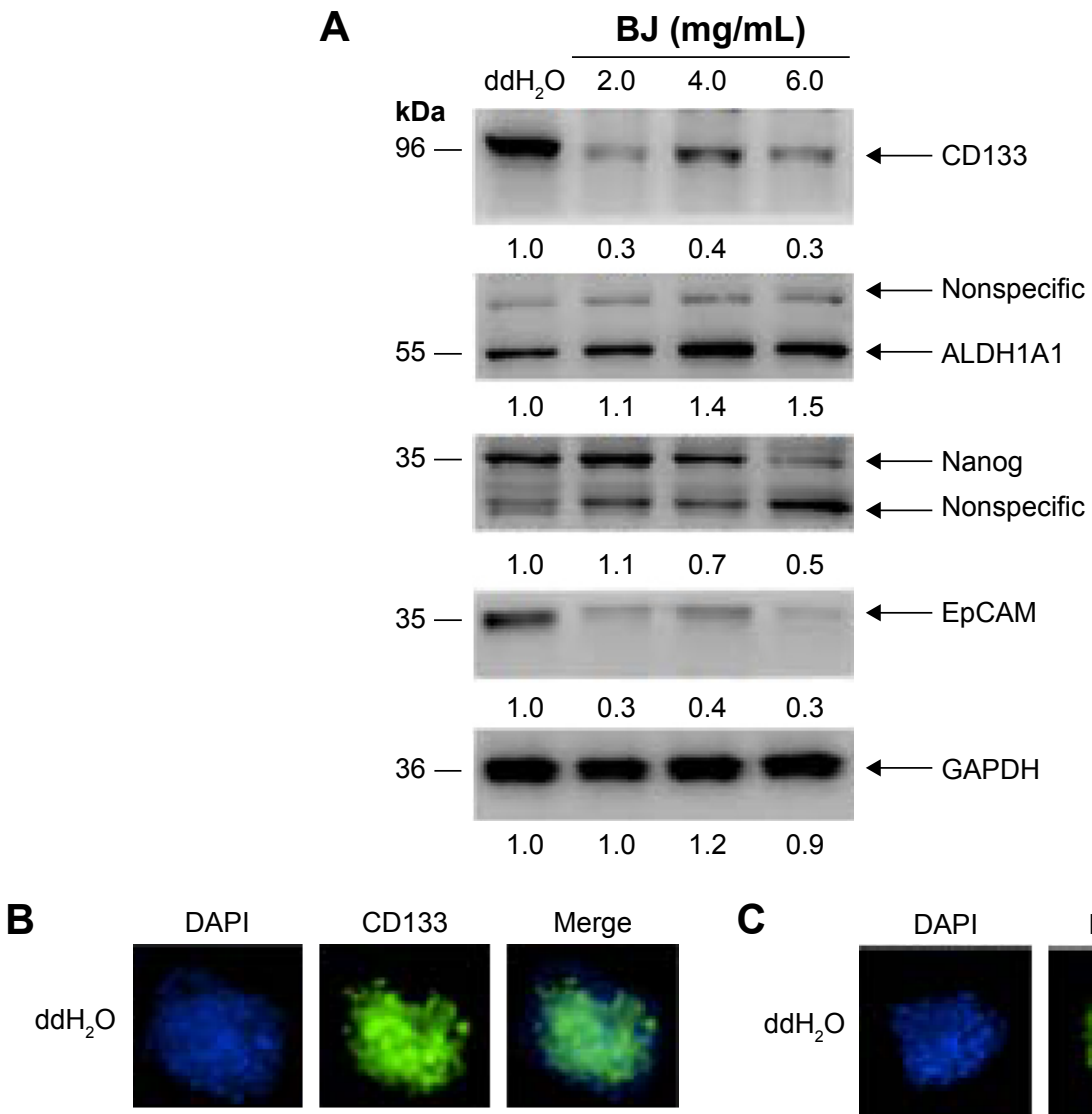

C
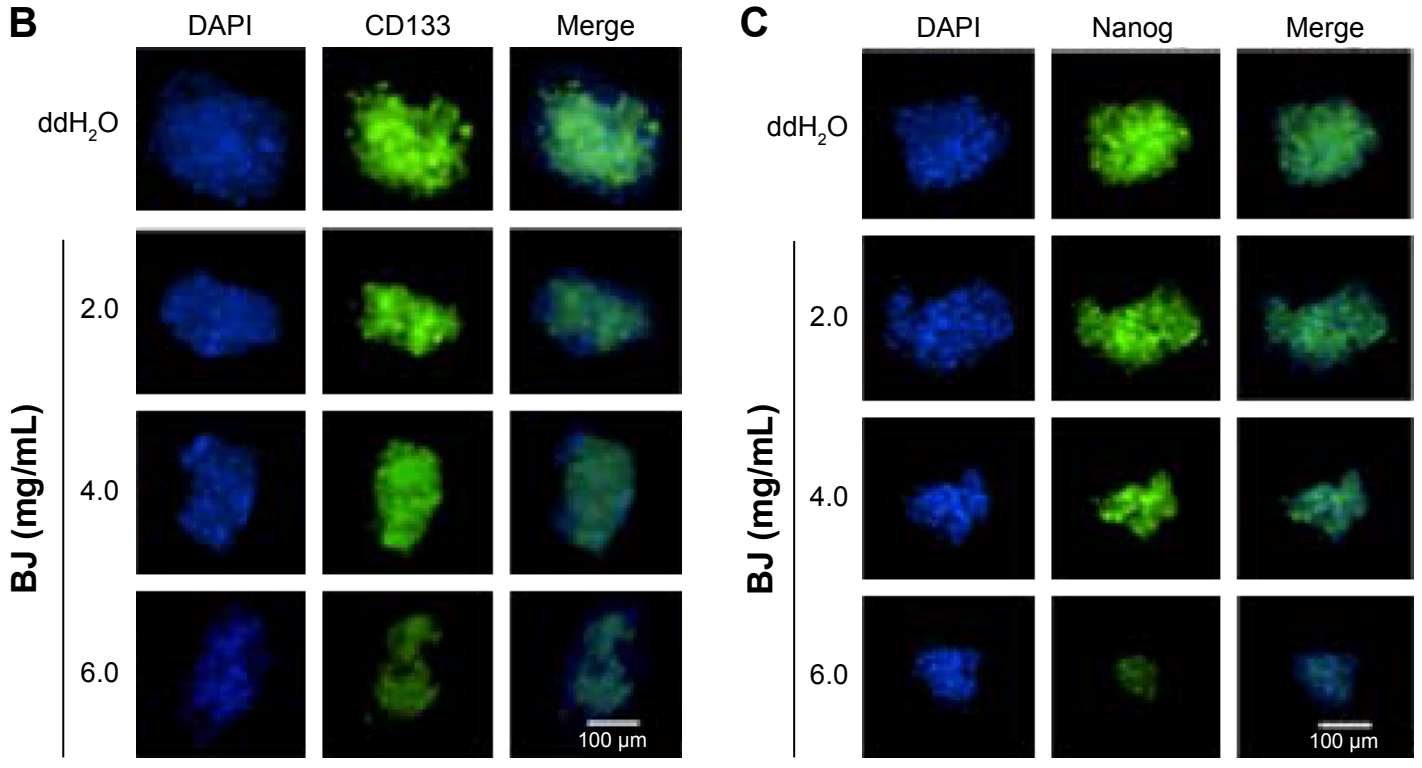

Figure 6 BJ reduced stemness marker of Hep3B spheres.

Notes: (A) Protein lysates from Hep3B spheroids treated with $2.0 \mathrm{mg} / \mathrm{mL}, 4.0 \mathrm{mg} / \mathrm{mL}$, and $6.0 \mathrm{mg} / \mathrm{mL}$ of BJ extract for 12 hours were resolved by SDS-PAGE gels and transferred to nitrocellulose membranes. The blots were analyzed for expressions of different stemness markers. They included Nanog, CDI33, EpCAM, and ALDHIAI with GAPDH as loading control. The values shown underneath each blot signify relative intensities compared with the results for vehicle control water treatment. (B) Merged images of immunofluorescent detection with CDI 33 antibody (green) of Hep3B spheroids after BJ treatment at $2.0 \mathrm{mg} / \mathrm{mL}, 4.0 \mathrm{mg} / \mathrm{mL}$, and $6.0 \mathrm{mg} / \mathrm{mL}$ of BJ for 24 hours after counterstaining with DAPI (blue) (scale bar $=100 \mu \mathrm{m}$ and applies to all images). (C) Merged images of immunofluorescent detection with Nanog antibody (green) of Hep3B spheroids after BJ treatment with counterstaining of DAPI (blue) (scale bar $=100 \mu \mathrm{m}$ and applies to all images).

Abbreviations: BJ, Brucea javanica; DAPI, 4',6-diamidino-2-phenylindole; GAPDH, glyceraldehyde 3-phosphate dehydrogenase; PAGE, polyacrylamide gel electrophoresis; SDS, sodium dodecyl sulfate.

serves as a potent initiator against liver cancer cells and the progenitor stem-like cells by targeting their intrinsic EGFR. Downregulated EGFRs were distinct in Hep3B cells in Western blot analysis and the diminished immunofluorescence of the enriched spheroids when exposed to BJ. The insignificant EGFR levels in HepG2 and Huh7 prevented the cells from insinuation by the aqueous extract.
Strategies aimed at efficient targeting of cancer stemlike cells are becoming more important to monitor the evolvement of cancer development and eradication. The identification of liver cancer CSC and its target destruction is challenging. CSCs are critical in initiating tumor progression, mediating metastasis and generating drug resistance. ${ }^{19}$ While cytotoxic treatments such as conventional 
A

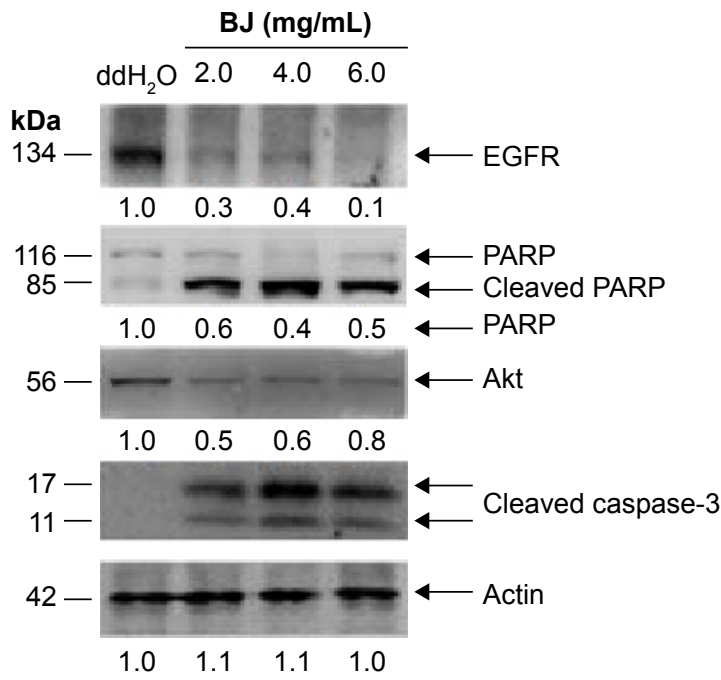

B

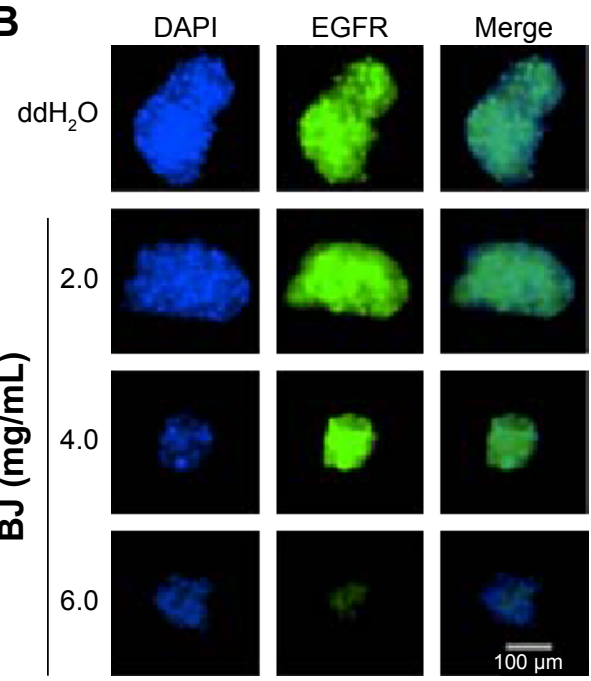

Figure 7 BJ increased apoptotic characteristics of Hep3B spheres.

Notes: (A) Protein lysates from Hep3B spheroids treated with $2.0 \mathrm{mg} / \mathrm{mL}, 4.0 \mathrm{mg} / \mathrm{mL}$, and $6.0 \mathrm{mg} / \mathrm{mL}$ of $B$ J extract for 12 hours were resolved and transferred. The blots were analyzed for expressions of PARP, Akt, and caspase-3 with GAPDH as loading control. The values shown underneath each plot signify relative intensities compared with the results of vehicle control water treatment. (B) Merged images of immunofluorescent detection with EGFR antibody (green) of Hep3B spheroids after BJ treatment for 24 hours and DAPI counterstaining (blue) (scale bar $=100 \mu \mathrm{m}$ and applies to all images).

Abbreviations: BJ, Brucea javanica; DAPI, 4',6-diamidino-2-phenylindole; EGFR, epidermal growth factor receptor; GAPDH, glyceraldehyde 3-phosphate dehydrogenase; PARP, poly(adenosine diphosphate-ribose) polymerase.

chemotherapy remove tumors by eradicating active tumor cells, the stem-like cancer cells survive and remain quiescent. More convincing evidence showed that common therapies indeed retain these stem-like subpopulations separated from original tumors and multiply. ${ }^{20}$ The findings underscored new alternative treatment strategies targeting stem cells that showed promise to improve patient response by prolonging survival rates. The identification by eradication of stem cells for aqueous CHM marks a significant stride in augmenting current target therapy.

\section{Conclusion}

Traditional Chinese medicinal herb BJ cured patients in East Asia and the nearby areas for years. The work proved that aqueous BJ extract promoted apoptosis in liver cancer cells. Moreover, the extract repressed the growth of the derived stem-like cells. The extended application of conventional CHM serves as an alternative measure and complements current available cancer therapy.

\section{Acknowledgments}

The work was supported by grants from the Ministry of Science and Technology, Executive Yuan, Republic of China (MOST 104-2320-B-003-001), and the National Taiwan Normal University (102T3040B2, 103T3040D2, and 104T3040C2). The authors acknowledge the Department of Life Science and Instrumentation Center, National Taiwan Normal University, for technical assistance of fluorescence and confocal laser microscopy.

\section{Disclosure}

The authors report no conflicts of interest in this work.

\section{References}

1. Wang X, Wang N, Cheung F, Lao L, Li C, Feng Y. Chinese medicines for prevention and treatment of human hepatocellular carcinoma: current progress on pharmacological actions and mechanisms. J Integr Med. 2015;13(3):142-164.

2. Wang H, Qian Z, Zhao H, et al. CSN5 silencing reverses sorafenib resistance of human hepatocellular carcinoma HepG2 cells. Mol Med Rep. 2015;12(3):3902-3908.

3. Ye QM, Bai LL, Hu SZ, et al. Isolation, chemotaxonomic significance and cytotoxic effects of quassinoids from Brucea javanica. Fitoterapia. 2015;105:66-72.

4. Zhao L, Li C, Zhang Y, Wen Q, Ren D. Phytochemical and biological activities of an anticancer plant medicine: Brucea javanica. Anticancer Agents Med Chem. 2014;14(3):440-458.

5. Nakao R, Mizukami C, Kawamura Y, et al. Evaluation of efficacy of bruceine A, a natural quassinoid compound extracted from a medicinal plant, Brucea javanica, for canine babesiosis. J Vet Med Sci. 2009;71(1): $33-41$.

6. Nie YL, Liu KX, Mao XY, Li YL, Li J, Zhang MM. Effect of injection of Brucea javanica oil emulsion plus chemoradiotherapy for lung cancer: a review of clinical evidence. J Evid Based Med. 2012;5(4):216-225.

7. Lau ST, Lin ZX, Zhao M, Leung PS. Brucea javanica fruit induces cytotoxicity and apoptosis in pancreatic adenocarcinoma cell lines. Phytother Res. 2008;22(4):477-486.

8. Liu J, Huang XE, Tian GY, et al. Phase II study on safety and efficacy of Yadanzi(R) (Javanica oil emulsion injection) combined with chemotherapy for patients with gastric cancer. Asian Pac J Cancer Prev. 2013;14(3):2009-2012.

9. Lu YY, Huang XE, Cao J, et al. Phase II study on Javanica oil emulsion injection (Yadanzi(R)) combined with chemotherapy in treating patients with advanced lung adenocarcinoma. Asian Pac J Cancer Prev. 2013; 14(8):4791-4794.

10. Yang J, Li S, Xie C, et al. Anti-inflammatory activity of ethyl acetate fraction of the seeds of Brucea javanica. J Ethnopharmacol. 2013; 147(2):442-446. 
11. Farazi PA, DePinho RA. Hepatocellular carcinoma pathogenesis: from genes to environment. Nat Rev Cancer. 2006;6(9):674-687.

12. Li Y, Martin RCG. Herbal medicine and hepatocellular carcinoma: applications and challenges. Evid Based Complement Alternat Med. 2011;2011:541209.

13. Xiao Z, Ching Chow S, Han Li C, et al. Role of microRNA-95 in the anticancer activity of Brucein D in hepatocellular carcinoma. Eur $J$ Pharmacol. 2014;728:141-150.

14. Yue Y, Yang Y, Shi L, Wang Z. Suppression of human hepatocellular cancer cell proliferation by Brucea javanica oil-loaded liposomes via induction of apoptosis. Arch Med Sci. 2015;11(4):856-862.

15. Leon G, MacDonagh L, Finn SP, Cuffe S, Barr MP. Cancer stem cells in drug resistant lung cancer: targeting cell surface markers and signaling pathways. Pharmacol Ther. 2016;158:71-90.
16. Shih J-Y, Gow C-H, Yang P-C. EGFR mutation conferring primary resistance to gefitinib in non-small-cell lung cancer. $N$ Engl J Med. 2005; 353(2):207-208.

17. Gao H, Lamusta J, Zhang W-F, et al. Tumor cell selective cytotoxicity and apoptosis induction by an herbal preparation from Brucea javanica. N Am J Med Sci (Boston). 2011;4(2):62-66.

18. Ghavami S, Hashemi M, Ande SR, et al. Apoptosis and cancer: mutations within caspase genes. J Med Genet. 2009;46(8):497-510.

19. Oishi N, Yamashita T, Kaneko S. Molecular biology of liver cancer stem cells. Liver Cancer. 2014;3(2):71-84.

20. Oh SJ, Noh KH, Lee YH, et al. Targeting stemness is an effective strategy to control EML4-ALK+ non-small cell lung cancer cells. Oncotarget. 2015;6(37):40255-40267.

\section{Publish your work in this journal}

Drug Design, Development and Therapy is an international, peerreviewed open-access journal that spans the spectrum of drug design and development through to clinical applications. Clinical outcomes, patient safety, and programs for the development and effective, safe, and sustained use of medicines are a feature of the journal, which has also been accepted for indexing on PubMed Central. The manuscript management system is completely online and includes a very quick and fair peer-review system, which is all easy to use. Visit http://www.dovepress.com/testimonials.php to read real quotes from published authors.

Submit your manuscript here: http://www.dovepress.com/drug-design-development-and-therapy-journal 